Dom. Cien., ISSN: 2477-8818

Vol. 3, núm. 4, octubre, 2017, pp. 485-508

\title{
Competencias lingüísticas y su incidencia en la pronunciación de inglés en estudiantes
}

\section{Linguistic competences and their incidence in the pronunciation of English in students}

Francisco D. Sandoval-Ortiz ${ }^{\text {I }}$

Universidad Central del Ecuador

Quito; Ecuador

fdsandoval@uce.edu.ec

dantefran82@gmail.com

\section{Marco V. Mier-Urbina II \\ Universidad Central del Ecuador \\ Quito; Ecuador \\ mvmier@uce.edu.ec \\ mvmu41@gmail.com}

\section{Edison A. Almachi-Montahuano III Universidad Central del Ecuador Quito; Ecuador eaalmachi@uce.edu.ec eddyalejandropucesi@gamail.com}

\author{
María G. Figueroa-Arellano IV \\ Universidad Central del Ecuador \\ Quito; Ecuador \\ mgfigueroa@uce.edu.ec \\ gabicita30@yahoo.com
}

\author{
Manuel W. Alvarado-Miles V \\ Universidad Central del Ecuador \\ Quito; Ecuador \\ mwalvarado@uce.edu.ec \\ alvaradowilson70@gmail.com
}

Recibido: 30 de enero de $2017 *$ Corregido: 20 de febrero de $2017 *$ Aceptado: 20 junio de 2017

I. Magister en Docencia Universitaria y Administración Educativa; Licenciado en Ciencias de la Educación Mención Ingles; Universidad Central del Ecuador.

II. Magister en Linguistica Aplicada a la enseñanza Bilingue Español-Inglés; Magister en Gerencia y Liderazgo Educacional; Licenciado en Ciencias de la Educación mención Ingles; Universidad Central del Ecuador.

III. Magister en Linguistica Aplicada a la Enseñanza Bilingue Español-Inglés; Licenciado en Ciencias de la Educación Mención Ingles; Técnico Superior en Análisis de Sistemas; Tecnólogo en Sistemas de Automatización; Universidad Central del Ecuador.

IV. Master of Science in Curriculum and Instruction, English as a Second Language; Ingeniera en Gestión Turística y Medio Ambiente; Guía de Turismo; Universidad Central del Ecuador.

v. Magister en Gerencia y Liderazgo Educacional; Licenciado en Ciencias de la Educación Mención Ingles; Tecnólogo En Administración de Empresas; Técnico Superior en Administración de Empresas; Universidad Central del Ecuador. 


\section{Resumen}

El objetivo principal de esta investigación es la propuesta de solución al problema de las limitadas Competencias Lingüísticas que inciden en la Pronunciación de inglés en los estudiantes de los primeros niveles de la Facultad de Ciencias Administrativas y Económicas, Escuela de Administración, Campus Sur de la Universidad Politécnica Salesiana de Quito. Las Competencias Lingüísticas (Escuchar, Hablar, Leer y Escribir) son importantes para el aprendizaje de inglés porque a través de ellas se llega a tener en cuenta la especificidad de la Lengua Oral y la Lengua Escrita. También, para lograr una buena comunicación en inglés se debe prestar atención a la Pronunciación de los sonidos (fonemas) y a la entonación y fluidez. Las interrogantes de investigación que surgen para proponer la alternativa de solución al problema se refieren al análisis del desarrollo de las Competencias Lingüísticas como punto clave para lograr un nivel óptimo de Pronunciación en inglés. Este trabajo de investigación se ha desarrollado de acuerdo a las pautas de la investigación crítico - propositiva y por tanto es de tipo cuanti - cualitativa. La principal fuente de información primaria son las encuestas aplicadas a estudiantes y docentes utilizando el Cuestionario como instrumento y los conocimientos establecidos en el desarrollo del Marco Conceptual. Su verificación estuvo respaldada mediante el análisis de expertos tanto en investigación, como en el área y temas investigados. En el presente trabajo de Investigación se concluye que el desarrollo de las Competencias Lingüísticas es bueno, pero no es completo y es limitado; y que el nivel de Pronunciación en inglés que los estudiantes tienen es bajo. Finalmente la propuesta de solución al problema demuestra ser de gran utilidad porque intenta orientar a que las Competencias Lingüísticas se desarrollen considerando como objetivo que el nivel de Pronunciación de los estudiantes mejore significativamente, permitiéndoles lograr una mejor comunicación.

Palabras clave: Competencias lingüísticas; pronunciación de inglés; enseñanza-aprendizaje; comunicación; alfabeto fonético internacional. 


\section{Abstract}

The main goal of this research is the proposed solution to the problem of the limited Language Skills and its incidence on the Pronunciation of English in the first level students from the Administrative and Financial science faculty of the Management School, south campus of the Salesian Polytechnic University of Quito. The Language skills (Listening, Speaking, Reading and Writing) are the English-learning base because through them, it is possible to take into account the specificity of Oral Language and Written Language. In addition, in order to fulfill a good communication in English, it must be taken into account the Pronunciation of Sounds (Phonemes); and the intonation and fluency which are part of the language. The questions for the research arise in order to propose the alternative of solution to the problem and they consider the analysis of the Language Skills development as a key point to fulfill an optimum level of Pronunciation in English. This research has been performed according to the norms of the critical-proactive research and that is the reason why it is quanti - qualitative. The principal source of primary information is the survey directed to students and docents using the Questionnaire as instrument and the established knowledge in the development of the Conceptual Framework. Its approval was supported by the analysis of experts in both research and the researched topics and area. In this research, it is concluded that the development of the Language Skills is good, but it is not complete and it is limited; and the level of Pronunciation in English the students have is low. Finally, the proposed solution to the problem proves to be very useful because it attempts to guide the Language Skills to be developed considering as the main goal, the significant improvement of the level of Pronunciation in English of the students, allowing them to fulfill a better communication.

Key words: Linguistic skills; teaching-learning; english pronunciation; communication; international phonetic alphabet. 


\section{Introducción.}

El presente trabajo de investigación se justifica porque no existe una propuesta alternativa para desarrollar las competencias lingüísticas en inglés en la Universidad Politécnica Salesiana y que incida directamente con su pronunciación y la originalidad que este trabajo encierra ayudará a los estudiantes a trabajar mejor y desarrollar sus competencias de escuchar, hablar, leer y escribir para comunicarse de una mejor manera en Inglés. (Rico Yate, Ramírez Montoya, \& Montiel Bautista, 2016) (Bygate, 1991)

El interés que este trabajo de investigación manifiesta se ve reflejado en el grupo docente y en los estudiantes de la Universidad Politécnica Salesiana porque cuentan con un material auténtico y ejercicios acordes al mismo, enfocados al desarrollo de las competencias lingüísticas en inglés y a su pronunciación y al mismo tiempo les ofrece diferentes actividades que están acordes a la realidad en la que se desenvuelven. (Rosales Bremont, Zarate Ortiz, \& Lozano Rodríguez,, 2013) (Durán Martínez \& Beltrán Llavador, 2012)

Este trabajo de investigación tiene una gran importancia porque establece bases para la adquisición y uso de un idioma inglés significativo para los estudiantes ya que para dominar las cuatro competencias del inglés, se debe empezar por la competencia de escuchar y continuar con las competencias de hablar, leer y escribir. (Korthagen, Loughran, \& Rusell, 2006)

Los beneficiarios directos de este trabajo de investigación serán los estudiantes, los cuales requieren conocer un idioma inglés que tenga relación con sus necesidades académicas, sociales y profesionales debido a que en este mundo globalizado, el inglés es utilizado en todos los aspectos 
que forman parte de su realidad y los ayuda a identificarse mejor. (Domínguez León \& Núñez Arévalo, 2012)

La utilidad teórica que el presente trabajo de investigación ofrecerá a los docentes comprenderá teoría relacionada a fonética y lingüística, vocabulario y gramática que ayudará a enseñar y explicar a los estudiantes cuantos sonidos fonéticos existen en el inglés y cómo suena cada uno de ellos para que los estudiantes tengan una base teórica que les permita mejorar sus competencias lingüísticas en inglés.

La utilidad práctica que este trabajo de investigación propone, considera situaciones reales en las que los estudiantes deberán desenvolverse y vocabulario que deberán usar apropiadamente, además se convertirá en un aporte útil para la labor profesional del docente, que contribuirá a mejorar el proceso de desarrollar las competencias del lenguaje con lo que se mejorará los resultados que se busca en los objetivos propuestos en la acción docente.

El presente estudio busca verificar la incidencia de las competencias lingüísticas en la pronunciación de Inglés en los estudiantes de los primeros niveles de la Facultad de Ciencias Administrativas y Económicas, Escuela de Administración, Campus Sur de la Universidad Politécnica Salesiana de Quito, en el período 40, año 2012.

\section{Metodología.}

La investigación, se basó en el enfoque crítico-propositivo, su accionar se dirigió a recabar información de la fuente y someterla a un análisis estadístico, para criticar la realidad existente y determinar la esencia del fenómeno causa-efecto con respaldo en su marco teórico para plantear una alternativa de solución asumiendo una realidad dinámica. Tuvo 
también un enfoque de carácter cuanti-cualitativo. Cuantitativa porque es normativa, explicativa y realista; y Cualitativa porque es participativa, humanista e interpretativa.

Población y Muestra

\begin{tabular}{|l|c|}
\hline Unidades de observación & No. \\
\hline Docentes & 6 \\
\hline Estudiantes & 120 \\
\hline TOTAL & $\mathbf{1 2 6}$ \\
\hline
\end{tabular}

Técnicas e Instrumentos de la Recolección de Información

Encuesta

Se utiliza la técnica de la encuesta, con el instrumento cuestionario, dirigido a profesores y estudiantes, con preguntas cerradas, que facilitan recoger la información de las variables que son el objeto de la investigación.

\section{Validez y confiabilidad}

La validez y confiabilidad de las encuestas a aplicar son analizadas por expertos tanto en investigación como del área y temas investigados, quienes emiten los respectivos juicios de valor sobre la validación, para su respectiva corrección de los instrumentos. 
Plan para la recolección de la información

\begin{tabular}{|l|l|}
\hline \multicolumn{1}{|c|}{ PREGUNTAS BÁSICAS } & \multicolumn{1}{c|}{ EXPLICACIÓN } \\
\hline 1. ¿Para qué? & Para solucionar el problema \\
\hline 2. ¿De qué personas? & Estudiantes, docentes \\
\hline 3. ¿Sobre qué aspectos? & $\begin{array}{l}\text { La variable independiente } \\
\text { (competencias lingǘsticas) y la variable } \\
\text { dependiente (pronunciación en inglés) }\end{array}$ \\
\hline 4. ¿Quiénes? & Investigador \\
\hline 5. ¿Cuándo? & Período 41, año lectivo 2012 \\
\hline 6. ¿Dónde? & Universidad Politécnica Salesiana \\
\hline 7. ¿Cuántas veces? & Dos veces (pilotaje - definitiva) \\
\hline 8. ¿Qué técnicas de recolección? & Encuestas \\
\hline 9. ¿Con qué? & Cuestionario \\
\hline 10. ¿En qué situación? & Normal \\
\hline
\end{tabular}

Cuadro $N^{o}$ 1.- Plan de recolección

\section{Plan para el Procesamiento y Análisis de la Información}

- Revisión crítica de la información recogida; es decir limpieza de información defectuosa: contradictoria, incompleta, no pertinente.

- Tabulación o cuadros según variables

- Estudio estadístico de datos para presentación de resultados 
- Análisis de los resultados estadísticos, destacando tendencias o relaciones fundamentales de acuerdo con los objetivos e interrogantes

- Interpretación de los resultados, con apoyo del marco teórico, en el aspecto pertinente.

- Establecimiento de conclusiones y recomendaciones.

\section{Resultados.}

\section{1.- ¿El docente propone temas de actualidad para lograr conversaciones en inglés?}

\begin{tabular}{|l|c|c|}
\hline Alternativas & Frecuencia & Porcentaje \\
\hline Siempre & 86 & $72 \%$ \\
Frecuentemente & 33 & $27 \%$ \\
Rara Vez & 0 & $0 \%$ \\
Nunca & 1 & $1 \%$ \\
\hline \multicolumn{1}{|c|}{ Total } & 120 & $100 \%$ \\
\hline
\end{tabular}

\section{Cuadro $N^{\circ}$ 1.- Temas de Actualidad}

El $72 \%$ y el $27 \%$ de estudiantes manifiestan que Siempre o Frecuentemente se proponen temas de actualidad para lograr conversaciones en inglés, pero el 1\% considera que Nunca se lo hace.

Mediante estos datos se aprecia que los estudiantes de la Universidad Politécnica Salesiana utilizan casi todo el tiempo temas de actualidad para conversar en inglés desarrollando su competencia de hablar, lo que demuestra que se da mucha prioridad a esta competencia. 


\section{2.- ¿El docente utiliza recursos de audio o video que le permitan a usted escuchar en inglés?}

\begin{tabular}{|l|c|c|}
\hline Alternativas & Frecuencia & Porcentaje \\
\hline Siempre & 79 & $66 \%$ \\
Frecuentemente & 35 & $29 \%$ \\
Rara Vez & 6 & $5 \%$ \\
Nunca & 0 & $0 \%$ \\
\hline \multicolumn{1}{|c|}{ Total } & 120 & $100 \%$ \\
\hline
\end{tabular}

\section{Cuadro $N^{\circ}$ 2.- Recursos de Audio o Video}

El 66\% y el 29\% de estudiantes consideran que Siempre o Frecuentemente se utiliza recursos de audio o video para escuchar en inglés, mientras que el 5\% piensan que Rara vez se lo hace.

Con estos datos se aprecia que los estudiantes de inglés de la Universidad Politécnica Salesiana utilizan la mayoría del tiempo recursos de audio y video logrando desarrollar su competencia de escuchar, demostrando así también, que el Audio es otra competencia a la cual se da mucha prioridad.

3.- ¿El docente provee artículos de lectura como periódicos, revistas, libros en inglés para que usted desarrolle su comprensión lectora?

\begin{tabular}{|l|c|c|}
\hline Alternativas & Frecuencia & Porcentaje \\
\hline Siempre & 46 & $38 \%$ \\
Frecuentemente & 49 & $41 \%$ \\
Rara Vez & 23 & $19 \%$ \\
Nunca & 2 & $2 \%$ \\
\hline \multicolumn{1}{|c|}{ Total } & 120 & $100 \%$ \\
\hline
\end{tabular}

Cuadro $N^{\circ}$ 3.- Comprensión Lectora 
El $38 \%$ y el $41 \%$ de estudiantes consideran que Siempre o Frecuentemente se provee artículos de lectura para desarrollar su comprensión lectora, mientras que el $19 \%$ y el $2 \%$ piensan que Rara vez o Nunca se lo hace.

Con estos datos se aprecia que los estudiantes de inglés utilizan artículos de lectura que les permiten desarrollar su comprensión lectora, sin embargo es una competencia que no se desarrolla plenamente.

\section{4.- ¿El docente aplica ejercicios en los cuales usted pueda desarrollar su escritura en inglés?}

\begin{tabular}{|l|c|c|}
\hline \multicolumn{1}{|c|}{ Alternativas } & Frecuencia & Porcentaje \\
\hline Siempre & 81 & $67 \%$ \\
Frecuentemente & 33 & $28 \%$ \\
Rara Vez & 6 & $5 \%$ \\
Nunca & 0 & $0 \%$ \\
\hline \multicolumn{1}{|c|}{ Total } & 120 & $100 \%$ \\
\hline
\end{tabular}

\section{Cuadro $N^{\circ}$ 4.- Escritura en Inglés}

El $67 \%$ y el $28 \%$ de estudiantes consideran que Siempre o Frecuentemente se aplica ejercicios para desarrollar su escritura en inglés, mientras que el 5\% piensan que Rara vez se lo hace.

De esta forma se entiende que los estudiantes de inglés de la Universidad Politécnica Salesiana la mayoría de las veces hacen ejercicios que desarrollan su competencia de escribir demostrando que la escritura es otra competencia que encierra mucha importancia en la enseñanza de inglés y a la cual se da prioridad. 


\section{5.- ¿El docente utiliza ejercicios en los cuales se empleen reglas de pronunciación en inglés?}

\begin{tabular}{|l|c|c|}
\hline \multicolumn{1}{|c|}{ Alternativas } & Frecuencia & Porcentaje \\
\hline Siempre & 72 & $60 \%$ \\
Frecuentemente & 40 & $33 \%$ \\
Rara Vez & 8 & $7 \%$ \\
Nunca & 0 & $0 \%$ \\
\hline \multicolumn{1}{|c|}{ Total } & 120 & $100 \%$ \\
\hline
\end{tabular}

Cuadro $N^{\circ}$ 5.- Pronunciación en Inglés

El $60 \%$ y el $33 \%$ de estudiantes consideran que Siempre o Frecuentemente se aplica ejercicios en los cuales se emplean reglas de pronunciación en inglés, pero el 7\% piensan que Rara vez se lo hace.

Mediante estos datos se aprecia que los estudiantes de inglés de la Universidad Politécnica Salesiana tienen un buen nivel de pronunciación, aunque podría mejorarse ya que solo la mitad de las veces Siempre se emplean ejercicios que les permitan tener una noción sobre la pronunciación en inglés.

\section{6.- ¿El docente hace un estudio del Alfabeto Fonético Internacional?}

\begin{tabular}{|l|c|c|}
\hline \multicolumn{1}{|c|}{ Alternativas } & Frecuencia & Porcentaje \\
\hline Siempre & 35 & $29 \%$ \\
Frecuentemente & 52 & $43 \%$ \\
Rara Vez & 27 & $23 \%$ \\
Nunca & 6 & $5 \%$ \\
\hline \multicolumn{1}{|c|}{ Total } & 120 & $100 \%$ \\
\hline
\end{tabular}

Cuadro $N^{\circ}$ 6.- Alfabeto Fonético Internacional 
El $29 \%$ y el $43 \%$ de estudiantes consideran que Siempre o Frecuentemente se hace un estudio sobre el Alfabeto Fonético Internacional, mientras que el 23\% y el 5\% piensan que Rara vez o Nunca se lo hace.

Mediante estos datos se entiende que los estudiantes conocen poco al Alfabeto Fonético Internacional; su estudio debe ser profundizado más y se lo debe incluir siempre que sea posible en el desarrollo de las cuatro competencias ya que un buen porcentaje considera no tener una idea clara sobre el tema.

7.- ¿El docente aplica ejercicios en los que se distinga y practique la entonación en una conversación?

\begin{tabular}{|l|c|c|}
\hline \multicolumn{1}{|c|}{ Alternativas } & Frecuencia & Porcentaje \\
\hline Siempre & 78 & $65 \%$ \\
Frecuentemente & 30 & $43 \%$ \\
Rara Vez & 10 & $8 \%$ \\
Nunca & 2 & $2 \%$ \\
\hline \multicolumn{1}{|c|}{ Total } & 120 & $100 \%$ \\
\hline
\end{tabular}

\section{Cuadro $N^{\circ}$ 7.- Entonación}

El $65 \%$ y el $43 \%$ de estudiantes consideran que Siempre o Frecuentemente se aplica ejercicios que permiten distinguir y practicar la entonación en una conversación, pero el $8 \%$ y el 2\% piensan que Rara vez o Nunca se lo hace.

De esta forma se entiende que la entonación en una conversación es considerada como parte fundamental en el estudio de inglés en la Universidad Politécnica Salesiana y que los estudiantes son 
capaces de diferenciar los enunciados, las exclamaciones y las interrogantes, aunque no completamente.

8.- ¿El docente maneja actividades en las que se practique el ritmo de pronunciación en una conversación?

\begin{tabular}{|l|c|c|}
\hline \multicolumn{1}{|c|}{ Alternativas } & Frecuencia & Porcentaje \\
\hline Siempre & 66 & $55 \%$ \\
Frecuentemente & 45 & $37 \%$ \\
Rara Vez & 9 & $8 \%$ \\
Nunca & 0 & $0 \%$ \\
\hline \multicolumn{1}{|c|}{ Total } & 120 & $100 \%$ \\
\hline
\end{tabular}

Cuadro $N^{\circ}$ 8.- Ritmo de Pronunciación

El $55 \%$ y el $37 \%$ de estudiantes consideran que Siempre o Frecuentemente se maneja actividades que permiten practicar el ritmo de pronunciación en una conversación, mientras que el $8 \%$ consideran que Rara vez se lo hace.

A través de estos datos se entiende que el ritmo de pronunciación en una conversación es importante en el estudio de inglés en la Universidad Politécnica Salesiana y que los estudiantes tienen la oportunidad de practicarlo la mayoría de las veces, sin embargo, su estudio debe llegar a ser más prioritario. 
9.- ¿Considera usted que el desarrollo de sus competencias lingüísticas influye de manera importante en su pronunciación de inglés?

\begin{tabular}{|l|c|c|}
\hline \multicolumn{1}{|c|}{ Alternativas } & Frecuencia & Porcentaje \\
\hline Siempre & 100 & $83 \%$ \\
Frecuentemente & 20 & $17 \%$ \\
Rara Vez & 0 & $0 \%$ \\
Nunca & 0 & $0 \%$ \\
\hline \multicolumn{1}{|c|}{ Total } & 120 & $100 \%$ \\
\hline
\end{tabular}

\section{Cuadro $N^{\circ}$ 9.- Competencias Lingüisticas influyen en Pronunciación}

El $83 \%$ y el 17\% de estudiantes consideran que Siempre o Frecuentemente el desarrollo de sus competencias lingüísticas influye de manera importante en su pronunciación de inglés.

Mediante estos datos se entiende que el constante desarrollo de las competencias lingüísticas tiene un efecto considerable en el nivel de pronunciación que los estudiantes tienen.

10.- ¿Cree usted que podría utilizar mejor sus competencias lingüísticas si se emplearan más ejercicios en el desarrollo de su pronunciación en inglés?

\begin{tabular}{|l|c|c|}
\hline \multicolumn{1}{|c|}{ Alternativas } & Frecuencia & Porcentaje \\
\hline Siempre & 89 & $74 \%$ \\
Frecuentemente & 31 & $26 \%$ \\
Rara Vez & 0 & $0 \%$ \\
Nunca & 0 & $0 \%$ \\
\hline \multicolumn{1}{|c|}{ Total } & 120 & $100 \%$ \\
\hline
\end{tabular}

Cuadro $N^{\circ}$ 10.- Competencias Lingüísticas y Pronunciación de inglés 
El $74 \%$ y el $26 \%$ de estudiantes consideran que Siempre o Frecuentemente se podría utilizar mejor las competencias lingüísticas si se emplearan más ejercicios en el desarrollo de su pronunciación en inglés.

De esta forma se aprecia que la correcta pronunciación de inglés es una herramienta que permite al estudiante aprender, practicar y dominar mejor las competencias lingüísticas de un idioma.

\section{Encuesta dirigida a docentes}

\section{1.- ¿Usted propone temas de actualidad para lograr conversaciones en inglés?}

\begin{tabular}{|l|c|c|}
\hline \multicolumn{1}{|c|}{ Alternativas } & Frecuencia & Porcentaje \\
\hline Siempre & 3 & $50 \%$ \\
Frecuentemente & 3 & $50 \%$ \\
Rara Vez & 0 & $0 \%$ \\
Nunca & 0 & $0 \%$ \\
\hline \multicolumn{1}{|c|}{ Total } & 6 & $100 \%$ \\
\hline
\end{tabular}

\section{Cuadro $N^{\circ}$ 11.- Temas de Actualidad}

El 50\% y el 3\% de docentes manifiestan que Siempre o Frecuentemente proponen temas de actualidad para lograr conversaciones en inglés.

Mediante estos datos se aprecia que los docentes de Inglés de la Universidad Politécnica Salesiana introducen casi todo el tiempo temas de actualidad para lograr que los estudiantes conversen en inglés y que de esta manera su competencia de hablar se desarrolle mejor. 
2.- ¿Usted utiliza recursos de audio o video que permitan a los estudiantes escuchar en inglés?

\begin{tabular}{|l|c|c|}
\hline \multicolumn{1}{|c|}{ Alternativas } & Frecuencia & Porcentaje \\
\hline Siempre & 4 & $67 \%$ \\
Frecuentemente & 2 & $33 \%$ \\
Rara Vez & 0 & $0 \%$ \\
Nunca & 0 & $0 \%$ \\
\hline \multicolumn{1}{|c|}{ Total } & 6 & $100 \%$ \\
\hline
\end{tabular}

\section{Cuadro $\mathbf{N}^{\circ}$ 12.- Recursos de Audio o Video}

El 67\% y el 33\% de docentes consideran que Siempre o Frecuentemente utilizan recursos de audio o video para hacer que los estudiantes escuchen en inglés.

Con estos datos se aprecia que los docentes de inglés de la Universidad Politécnica Salesiana logran que los estudiantes ocupen la mayoría del tiempo recursos de audio y video los cuales les permiten desarrollar su competencia de escuchar.

3.- ¿Usted provee artículos de lectura como periódicos, revistas, libros para que los estudiantes desarrollen su comprensión lectora en inglés?

\begin{tabular}{|l|c|c|}
\hline \multicolumn{1}{|c|}{ Alternativas } & Frecuencia & Porcentaje \\
\hline Siempre & 1 & $16 \%$ \\
Frecuentemente & 3 & $50 \%$ \\
Rara Vez & 1 & $17 \%$ \\
Nunca & 1 & $17 \%$ \\
\hline \multicolumn{1}{|c|}{ Total } & 6 & $100 \%$ \\
\hline
\end{tabular}

Cuadro $N^{\circ}$ 13.- Comprensión Lectora 
El $16 \%$ y el $50 \%$ de docentes consideran que Siempre o Frecuentemente proveen artículos de lectura para desarrollar comprensión lectora, mientras que un 17\% consideran que Rara vez y otro $17 \%$ que Nunca lo hacen.

Con estos datos se aprecia que los docentes de inglés de la Universidad Politécnica Salesiana la mayoría de las veces utilizan artículos de lectura que les permiten a los estudiantes desarrollar su comprensión lectora, y aunque se lo hace frecuentemente, todavía existe cierta falencia que se debería mejorar.

\section{4.- ¿Usted aplica ejercicios en los cuales los estudiantes puedan desarrollar su escritura en} inglés?

\begin{tabular}{|l|c|c|}
\hline \multicolumn{1}{|c|}{ Alternativas } & Frecuencia & Porcentaje \\
\hline Siempre & 2 & $34 \%$ \\
Frecuentemente & 2 & $33 \%$ \\
Rara Vez & 2 & $33 \%$ \\
Nunca & 0 & $0 \%$ \\
\hline \multicolumn{1}{|c|}{ Total } & 6 & $100 \%$ \\
\hline
\end{tabular}

\section{Cuadro $N^{\circ}$ 14.- Escritura en Inglés}

Un $34 \%$ y otro $33 \%$ de docentes consideran que Siempre o Frecuentemente aplican ejercicios que permiten a los estudiantes desarrollar su escritura en inglés, mientras que el $33 \%$ piensan que Rara vez lo hacen.

De esta forma se considera que los docentes de inglés de la Universidad Politécnica Salesiana la mayoría de las veces hacen ejercicios que logran que el estudiante desarrolle su 
competencia de escribir demostrando que la escritura es otra competencia que encierra mucha importancia en la enseñanza de inglés.

\section{5.- ¿Usted utiliza ejercicios en los cuales se empleen reglas de pronunciación en inglés?}

\begin{tabular}{|l|c|c|}
\hline \multicolumn{1}{|c|}{ Alternativas } & Frecuencia & Porcentaje \\
\hline Siempre & 0 & $0 \%$ \\
Frecuentemente & 2 & $34 \%$ \\
Rara Vez & 2 & $33 \%$ \\
Nunca & 2 & $33 \%$ \\
\hline \multicolumn{1}{|c|}{ Total } & 6 & $100 \%$ \\
\hline
\end{tabular}

\section{Cuadro $N^{\circ}$ 15.- Pronunciación en Inglés}

El 34\% de docentes consideran que Frecuentemente aplican ejercicios para emplear reglas de pronunciación en inglés, mientras que un 33\% creen que Rara vez y otro $33 \%$ piensan que Nunca lo hacen.

Mediante estos datos se aprecia que los docentes de inglés de la Universidad Politécnica Salesiana no se preocupan mucho por lograr un buen nivel de pronunciación en los estudiantes ya que la frecuencia con que se trabaja este tema es baja y esto eventualmente se evidencia en el pobre nivel de pronunciación que los estudiantes tienen. 


\section{6.- ¿Usted hace un estudio del Alfabeto Fonético Internacional?}

\begin{tabular}{|l|c|c|}
\hline \multicolumn{1}{|c|}{ Alternativas } & Frecuencia & Porcentaje \\
\hline Siempre & 0 & $0 \%$ \\
Frecuentemente & 1 & $17 \%$ \\
Rara Vez & 3 & $50 \%$ \\
Nunca & 2 & $33 \%$ \\
\hline \multicolumn{1}{|c|}{ Total } & 6 & $100 \%$ \\
\hline
\end{tabular}

\section{Cuadro $N^{\circ}$ 16.- Alfabeto Fonético Internacional}

El $17 \%$ de docentes consideran que Frecuentemente se hace un estudio sobre el Alfabeto Fonético Internacional, mientras que el 50\% y el 33\% piensan que Rara vez o Nunca se lo hace.

Mediante estos datos se entiende que un estudio sobre el Alfabeto Fonético Internacional no se lo considera relevante en el proceso de enseñanza-aprendizaje del idioma inglés por parte de los docentes y consecuentemente los estudiantes no conocen esta importante herramienta para mejorar su nivel de pronunciación.

7.- ¿Usted aplica ejercicios en los que se distinga y practique la entonación en una conversación?

\begin{tabular}{|l|c|c|}
\hline \multicolumn{1}{|c|}{ Alternativas } & Frecuencia & Porcentaje \\
\hline Siempre & 0 & $0 \%$ \\
Frecuentemente & 2 & $33 \%$ \\
Rara Vez & 3 & $50 \%$ \\
Nunca & 1 & $17 \%$ \\
\hline \multicolumn{1}{|c|}{ Total } & 6 & $100 \%$ \\
\hline
\end{tabular}

Cuadro $N^{\circ}$ 17.- Entonación 


\section{8.- ¿Usted maneja actividades en las que se practiquen el ritmo de pronunciación en una} conversación?

\begin{tabular}{|l|c|c|}
\hline \multicolumn{1}{|c|}{ Alternativas } & Frecuencia & Porcentaje \\
\hline Siempre & 0 & $0 \%$ \\
Frecuentemente & 3 & $50 \%$ \\
Rara Vez & 3 & $50 \%$ \\
Nunca & 0 & $0 \%$ \\
\hline \multicolumn{1}{|c|}{ Total } & 6 & $100 \%$ \\
\hline
\end{tabular}

Cuadro $N^{\circ}$ 18.- Ritmo de Pronunciación

El 50\% de docentes consideran que Frecuentemente manejan actividades en las que los estudiantes practican el ritmo de pronunciación en una conversación, mientras que el 50\% piensan que Rara vez lo hacen.

A través de estos datos se entiende que el ritmo de pronunciación en una conversación se lo considera como parte importante en el estudio de inglés, pero que de todas maneras la frecuencia con que se realiza el estudio no es la mejor.

9.- ¿Considera usted que el desarrollo de las competencias lingǘsticas en los estudiantes influye de manera importante en su pronunciación de inglés? 


\begin{tabular}{|l|c|c|}
\hline \multicolumn{1}{|c|}{ Alternativas } & Frecuencia & Porcentaje \\
\hline Siempre & 4 & $67 \%$ \\
Frecuentemente & 2 & $33 \%$ \\
Rara Vez & 0 & $0 \%$ \\
Nunca & 0 & $0 \%$ \\
\hline \multicolumn{1}{|c|}{ Total } & 6 & $100 \%$ \\
\hline
\end{tabular}

\section{Cuadro $N^{\circ}$ 19.- Competencias Lingüísticas influyen en Pronunciación}

El $67 \%$ y el $33 \%$ de docentes consideran que Siempre o Frecuentemente el desarrollo de las competencias lingüísticas en el estudiante influye de manera importante en su pronunciación de inglés.

Mediante estos datos se entiende que el constante desarrollo de las competencias lingüísticas tiene un efecto considerable en el nivel de pronunciación que los estudiantes tienen.

10.- ¿Cree usted que los estudiantes podrían utilizar mejor sus competencias lingüísticas si se emplearan más ejercicios en el desarrollo de su pronunciación en inglés?

\begin{tabular}{|l|c|c|}
\hline \multicolumn{1}{|c|}{ Alternativas } & Frecuencia & Porcentaje \\
\hline Siempre & 5 & $83 \%$ \\
Frecuentemente & 0 & $0 \%$ \\
Rara Vez & 1 & $17 \%$ \\
Nunca & 0 & $0 \%$ \\
\hline \multicolumn{1}{|c|}{ Total } & 6 & $100 \%$ \\
\hline
\end{tabular}

Cuadro $N^{\circ}$ 20.- Competencias Lingüísticas y Pronunciación de inglés 
El $83 \%$ de docentes consideran que Siempre o Frecuentemente los estudiantes podrían trabajar mejor sus competencias lingüísticas si se emplean más ejercicios en el desarrollo de su pronunciación en inglés, mientras que el $17 \%$ consideran que no es un factor muy relevante.

De esta forma se aprecia que la correcta pronunciación de inglés es una herramienta que le permite al estudiante aprender, practicar y dominar mejor las competencias lingüísticas de un idioma.

\section{Conclusiones.}

En el trabajo de investigación sobre "Competencias lingüísticas y su incidencia en la pronunciación de inglés en los estudiantes de los primeros niveles de la Facultad de Ciencias Administrativas y Económicas de la Escuela de Administración, Campus Sur de la Universidad Politécnica Salesiana de Quito, en el período 40", Se desarrolló una investigación con un enfoque crítico-propositivo y cuanti-cualitativo, la cual estableció información que fue analizada y representada en gráficos. Al finalizar el trabajo se establecen las siguientes Conclusiones y Recomendaciones.

El desarrollo que tienen las Competencias Lingüísticas en la Universidad Politécnica Salesiana de Quito, Campus Sur, no es el mejor ya que como se demuestra en la investigación, los estudiantes de la Facultad de Ciencias Administrativas y Económicas de la Escuela de Administración consideran que no siempre disponen de oportunidades para usar sus conocimientos de escuchar, hablar, leer o escribir y consideran también que un desarrollo más óptimo de sus Competencias Lingüísticas les permitirá ser participes de una más eficiente utilización del lenguaje. 
El nivel de pronunciación que tienen los estudiantes de la Facultad de Ciencias Administrativas y Económicas de la Escuela de Administración, en la Universidad Politécnica Salesiana de Quito, Campus Sur, es muy bajo debido a que la frecuencia con que se realizan ejercicios que promuevan la utilización del Alfabeto Fonético Internacional o que enfaticen en la importancia de la Fluidez Verbal o la Entonación al momento de transmitir una idea en inglés es considerablemente baja y además queda establecido que los estudiantes creen que el desarrollo y utilización de sus Competencias Lingüísticas mejoraría si se emplearan más ejercicios en el perfeccionamiento de su pronunciación en inglés.

La elaboración de una propuesta alternativa de solución al problema de las limitadas competencias lingüísticas que inciden en la pronunciación de inglés en los estudiantes de los primeros niveles de la Facultad de Ciencias Administrativas y Económicas, Escuela de Administración, Campus Sur de la Universidad Politécnica Salesiana de Quito es posible debido a que los estudiantes consideran que el desarrollo de sus Competencias Lingüísticas influyen de manera importante en su pronunciación de inglés y que el progreso de su nivel de pronunciación, les permitirá utilizar mejor sus Competencias Lingüísticas.

\section{Bibliografía.}

Bygate, M. (1991). Speaking. United Kingdom: Oxford University Press.

Domínguez León, A., \& Núñez Arévalo, M. (2012). La comprensión lectora en Inglés en la carrera de Medicina Veterinaria y Zootecnia. Revista Electrónica de Veterinaria, 4, 1-12.

Durán Martínez, R., \& Beltrán Llavador, F. (2012). Nuevos modelos de formación del profesorado de inglés: El caso de Castilla y León. Revista de Currículum y Formación de Profesorado, 17(3), 307-323.

Korthagen, F., Loughran, J., \& Rusell, T. (2006). Developing fundamental principles for teacher education programs and practices. Teaching and Teacher Education, 22(1), 1020-1041. 
Rico Yate, J., Ramírez Montoya, M., \& Montiel Bautista, S. (2016). Desarrollo de la competencia oral del inglés medianterecursos educativos abiertos. Apertura, 8(1), 1-15.

Rosales Bremont, B., Zarate Ortiz, J., \& Lozano Rodríguez,, A. (2013). Desarrollo de la competencia comunicativa en el idioma inglés en una plataforma interactiva. Revista Electrónica Sinéctica, 41(1), 1-11. 